\title{
RENAL VENOUS THROMBOSIS AND INFARCTION IN THE NEWBORN
}

\author{
BY \\ J. EDGAR MORISON, M.D., B.Sc. \\ From the Department of Pathology, Queen's University, Belfast, and the Belfast City
Hospital, Northern Ireland
}

Haemorrhagic infarction of the kidney is usually considered to be secondary to venous thrombosis and to be a rare lesion both in the newborn and in older children.

Most recent reports have described one (Heller, 1923; Aschner, 1927 ; Nordwall, 1933; Hepler, 1934; Marshall and Whapham, 1936; Behr, 1938); two (Petramand, 1923) or three (Oppenheim, 1920) cases occurring in the first year of life, and eight of these occurred in the neonatal period. A few of the older European workers found the condition more frequently and this was discussed by Oppenheim and by Faerber and Bussel (1930). The condition is, indeed, not rare and Cruickshank (1930) found fifteen examples among thirty cases of visceral thrombosis encountered in 800 neonatal deaths. Barenberg, Greenstein, Levy and Rosenbluth (1941) had five cases in the newborn in twenty-five autopsies in epidemics of diarrhoea complicated by secondary infections. Campbell (1937) noted eighteen cases under six months in the records of one hospital. Later he reported (Campbell and Matthews, 1942) two unilateral cases treated by nephrectomy. He did not distinguish cases with and without pyelonephritis. Pyelonephritis was responsible for the four cases reported by Schröder (1926). No detailed description of the lesions was given by Cruickshank or by Campbell. Oppenheim and Schröder have both discussed the pathology of special varieties of the condition in infancy and Behr has studied renal venous thrombosis at all ages, but no representative series of cases in the newborn has been studied.

It may be useful to describe eighteen cases without pyelonephritis. All but one of these occurred in the neonatal period. Four cases of pyelonephritis with vascular lesions, two of them in the neonatal period, will also be discussed. In all of these cases a detailed histological examination was made of nearly every organ of the body.

All cases of renal venous thrombosis are not associated with infarction and in some cases of haemorrhagic necrosis there is no venous thrombosis. It is proposed to distinguish three main groups.

(1) Thrombosis of radicles of renal veins.

(i) without infarction;

(ii) with infarction.

(2) Haemorrhagic infarction without primary renal venous thrombosis.

(3) Infarction secondary to renal inflammation with or without venous thrombosis.

Thrombosis of radicles of renal veins

Without infarction. In five cases there were thrombi in radicles of the renal veins without haemorrhage or damage to renal parenchyma.

Case 1. A male infant weighing $7 \mathrm{lb} .9 \mathrm{oz}$. $(3,430$ gm.) at birth lost $11 \mathrm{oz}$. (312 gm.) in the first three days of life and a further $12 \mathrm{oz}$. (340 gm.) by the seventh day. Icterus was then in excess of the normal, and diarrhoea and vomiting commenced and continued for four days. Blood-tinged fluid was obtained on lumbar puncture on the eleventh day. Diarrhoea and vomiting began again on the fifteenth day and continued until death on the nineteenth day when the weight was $5 \mathrm{lb} .7 \mathrm{oz}$. $(2,466$ $\mathrm{gm}$.).

Anatomical summary. Unhealed umbilical site: slight arteritis: scars in intima of umbilical vein. Thrombi in intrarenal veins with organization: old and recent emboli in small pulmonary arteries: terminal lung infarcts. Thrush stomatitis and oeso-

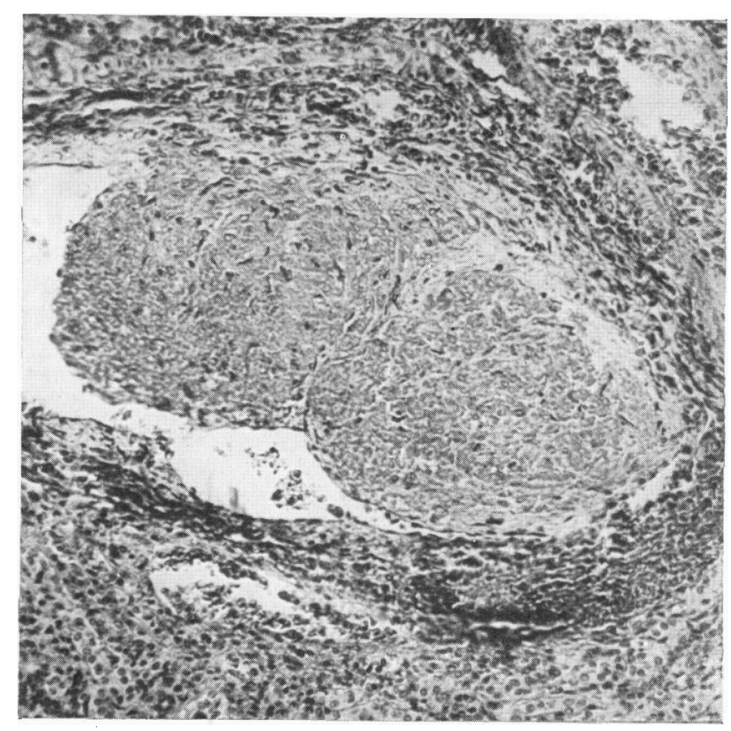

Fig. 1.-Organization of a thrombus at the commencement of an interlobar vein. Case 1. van Gieson. $\times 125$. 
phagitis. Bilateral purulent otitis media. Terminal pneumonia. Minimal subarachnoid haemorrhage.

In the intrarenal veins the thrombi were of different ages and some channels were not involved. The oldest thrombi were in dilated arcuate vessels and at the beginning of the interlobar veins, and here they were hyalinized and projected into the lumen, but were covered by endothelium and partly organized by fibrous tissue (fig. 1). The large and illsupported veins at the hilum were also dilated and columns and laminae of platelet thrombus supported a fibrin mesh with entangled red blood cells. Here there was no growth of endothelium over the thrombus or proliferation of fibrous tissue from the vein wall. In the small pulmonary arteries there were numerous hyalinized thrombi and sometimes endothelium had grown over these from the vessel wall (fig. 2). Groups of alveoli with indistinct walls

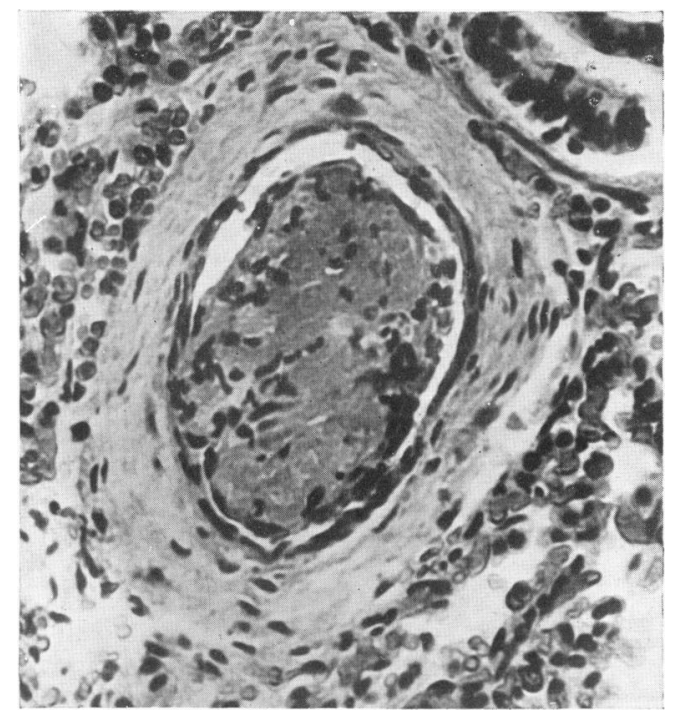

FIG. 2.-An embolic thrombus in a small pulmonary artery with endothelium growing over it. Case 1. H. E. $\times 275$.

were packed with red blood cells with no excess of polymorphonuclear leucocytes.

Case 2. A male child died suddenly on the seventeenth day. He had had an oral thrush infection from the twelfth day and was dehydrated. Extensive thrombi in the arcuate and small interlobar veins of the left kidney sometimes showed an endothelial covering and there were fragments of embolic thrombus in the pulmonary arteries.

Case 3. A male child died on the twenty-first day from broncho-pneumonia. There was a history of skin sepsis, episodes of diarrhoea and vomiting and a loss of weight from $9 \mathrm{lb}$. $(4,080 \mathrm{gm}$.) to 6 lb. 4 oz. $(2,835 \mathrm{gm}$.). In both kidneys about a quarter of the arcuate veins contained organizing thrombus, but the only reaction was a few mononuclear cells, some of which contained haemosiderin, near the vessels.

Cases 4 and 5. In two male infants aged twelve and fourteen days well formed thrombi were present without cellular reaction in the arcuate and interlobar veins of grossly congested kidneys. No other lesions were found in these infants, but their progress had never been satisfactory and they probably suffered from a septicaemia.

In the first three cases there was a clinical history of episodes of dehydration and septic lesions were present somewhere in the body. In cases 4 and 5 no septic lesions were found and severe dehydration was a terminal event. Thrombi were seen at postmortem in cases 1, 2 and 4, focal congestion of kidney tissue was noted in cases 1 and 4, and, except in case 2, the lesions were bilateral. Cases 1,2 and 3 provided evidence that the oldest thrombi, those showing most organization or endothelial overgrowth, were to be found in the arcuate veins. In no case was there infiltration of the peri-vascular, interstitial or peri-pelvic tissues or invasion of the lumen of the tubules by inflammatory cells. Other cases have been seen with thrombi in the intrarenal veins, but without cellular reaction or distinctive arrangement of platelets and fibrin. Thrombosis was probably agonal, but there was sometimes localized congestion of the kidney.

With infarction. In five cases red blood cells had escaped between the tubules of a few renal papillae and here epithelial cells were degenerating. In two cases extensive haemorrhage had enlarged the kidneys to twice their normal size and converted portions of them into little more than blood filled cysts, and in two others there were pale grayish-pink infarcts as well as haemorrhagic areas.

Case 6. A male infant was very asphyxiated after a difficult forceps delivery. He was fretful for three days and slightly jaundiced. On the fourth day the temperature was $100^{\circ} \mathrm{F}$., vomiting became severe, both ear drums were injected, and the weight had fallen from $6 \mathrm{lb} .13 \mathrm{oz}$. $(3,090 \mathrm{gm}$.) to $5 \mathrm{lb} .12 \mathrm{oz}$. $(2,610 \mathrm{gm}$.). On the fifth day the respirations were rapid and there were crepitations over the chest. He died on the seventh day.

Anatomical summary. Excessive deposits of debris of liquor amnii in alveoli: diffuse atelectasis: bilateral broncho-pneumonia: gross congestion all organs. Endothelialized thrombi in arcuate and interlobar veins of both kidneys and slight haemorrhage into medulla of left. Right catarrhal otitis media.

Urine was obtained at autopsy and contained only a few red blood cells.

Cases 7, 8, 9 and 10. These full term infants, two male and two female, died on the eighth, ninth, ninth and twenty-fourth days of life. Case 7 had a thrush infection and a terminal aspiration pneumonia. The mother of case 8 had a pre-eclamptic toxaemia and he developed a temperature of $102^{\circ} \mathrm{F}$. on the seventh day, but no cause for this was found. Case 9 had an unhealthy umbilicus and liver lesions and was described as case 6 in a paper on acute interstitial hepatitis (Morison, 1944). Case 10 had had skin sepsis for five days. All presented evidence of dehydration for at least two days before death. Only a little haemorrhage disrupted the pattern of the tubules towards the tip of a few pyramids, but the kidneys were enlarged and congested and their pelves were also congested. Poorly formed thrombi in the larger veins at the hilum showed no cellular 
reaction, but thrombi in the smaller intrarenal vessels were becoming covered with endothelium in cases 8 and 10 and organized in case 9 . Emboli were found in some pulmonary arteries in cases 9 and 10 and small pulmonary infarcts had developed in cases 7 and 8 .

Case 11. A male infant was admitted on the seventh day with a history of persistent crying. A lump was palpable in the right side of the abdomen and a little blood-stained urine was passed for the remaining three days of life.

Anatomical summary. Thrombi, partly covered by proliferation of endothelium, in intra-renal veins of both kidneys: haemorrhagic infarction of both kidneys: multiple emboli in pulmonary arteries.

Case 12. A female infant was born with multiple deformities. Feeding was difficult and by the twelfth day she was very ill, dehydrated and cyanosed and died the next day.

Anatomical summary. Gross atelectasis both lungs. Gross congestion all organs. Thrombi in intrarenal veins of both kidneys and organization of thrombi in a few arcuate veins: extensive haemorrhagic infarction of both kidneys: occasional emboli in pulmonary arteries. Cleft palate and multiple osseous deformities. Post-mortem blood urea $135 \mathrm{mgm}$. per $100 \mathrm{c.cm}$.

Much of the thrombus in the dilated intrarenal veins was recent, but after searching numerous sections hyalinized thrombus was found organized to the wall of an arcuate vein. In this and in the preceding case red blood cells had escaped, especially into the medulla, to produce lakes of blood with islands of degenerating tubules, and the kidneys were enlarged to over twice their normal size.

Case 13. This baby died on the ninth day with a severe thrush and mixed bacterial infection of mouth, pharynx, larynx and oesophagus and a bilateral pneumonia. He showed well organized thrombi in the intrarenal veins and a few small pale infarcts in the medulla were surrounded by extensive red infarcts. More terminal thrombus lay in the aorta and in the beginning of the renal arteries. The case was discussed as case 3 in a paper on thrombosis of the aorta (Morison, 1945).

Case 14. A male infant had developed thrush stomatitis, vomiting, severe diarrhoea and an abnormal loss of weight by the fifth day. Gentian violet was applied to the mouth and sulphadiazine given orally. The diarrhoea became less severe, but the temperature remained around $101-102^{\circ} \mathrm{F}$. and he drank poorly. On the eighth day the urine was blood stained, the abdomen became tense and rigid, diarrhoea persisted and he died the next day.

Anatomical summary. Thrush and heavy mixed bacterial infection of mouth, pharynx and oesophagus: laryngitis with ulceration : bilateral bronchopneumonia. Organizing thrombi in intrarenal veins of both kidneys with extensive red and pale venous infarcts. Post mortem blood urea $180 \mathrm{mgm}$. per $100 \mathrm{c.cm}$.

The kidneys were about twice their normal size. Large areas of necrosis occupied the medulla and extended with an irregular margin to involve the greater part of the cortex. In the left kidney these were dark red, but a few were paler and surrounded by dark red zones. In the right kidney (fig. 3) many areas were a grayish pink and contrasted better with the red zones around them. Histologically thrombi were organizing in many of the arcuate

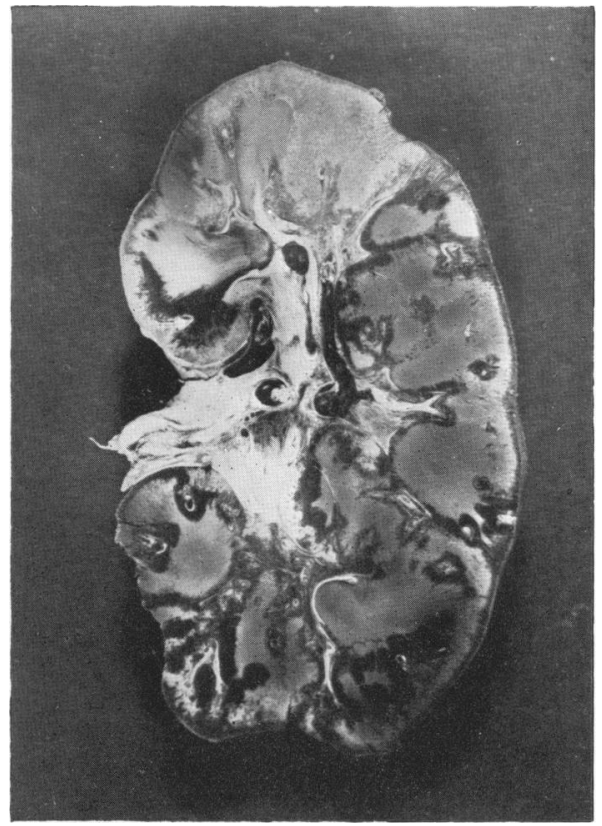

and in the smaller interlobar veins. In the red areas the tubules were separated from one another by extravasated red blood cells and, though the nuclei of the epithelial cells were distinct, the cytoplasm was disintegrating. In the pale infarct areas the outlines of the shrunken renal structures were indistinct, nuclei, if present, were pyknotic and red blood cells lying between the tubules were colourless, ill-defined discs. Various transitions existed between the red and pale infarct areas. In the cortex the sub-capsular blood vessels were not congested. Beyond the gross congestion marking the limits of the infarcts and intermingled with surviving glomeruli and other parenchymatous elements many of the convoluted tubules had lost all cellular detail and were eosinophilic. There were no fibrin thrombi in glomerular capillaries and the arteries were nowhere thrombosed.

These cases were often associated some days before death with conditions productive of dehydration. Endothelial cells were found on the surface of some of the thrombi in the smaller intrarenal veins in cases $6,8,10$ and 11 , and similar thrombi were invaded by fibroblasts in cases $9,12,13$ and 14 . No comparable reaction was present in the larger interlobar veins or in the veins at the hilum. Congenital atelectasis was found in cases 6, 10 and 12 and venous congestion of the viscera was excessive in cases 6, 11 and 12, but was of uncertain duration. Emboli in the pulmonary arteries or infarcts in the lungs were found in seven cases (cases 7-13).

\section{Haemorrhagic infarction without primary venous thrombosis}

Ante-mortem thrombi were not found in the veins in these cases, but blood had escaped from over- 
distended capillary channels and parenchymatous elements, especially in the medulla, were surrounded by confluent haemorrhages and undergoing necrosis.

Case 15. A male infant died $14 \frac{1}{2}$ hours after birth.

Anatomical summary. Erythroblastosis foetalis. Rhabdomyomatosis left ventricle. Patchy atelectasis: gross congestion all organs. Haemorrhagic infarction both kidneys. Bilateral hare lip and supernumerary digits.

Case 16. A male infant after a normal fall of birth weight started to lose weight from the seventh day. There was vomiting on the ninth day and sulphathiazole therapy was commenced. Severe vomiting and diarrhoea occurred on the tenth and death on the eleventh day.

Anatomical summary. Thrush infection with heavy mixed bacterial infection mouth, pharynx, larynx and oesophagus: acute rhinitis: catarrhal otitis media. Excessive deposits of amniotic debris in lung alveoli: patchy atelectasis: terminal pneumonia. Bilateral haemorrhagic infarction both kidneys.

Case 17. A female infant was jaundiced and slightly febrile on the fourth day and was treated with sulphathiazole. By the ninth day the clinical condition had improved and chemotherapy was stopped. Three days later the temperature rose again, convulsions occurred and the baby became cyanosed with rapid breathing and died the next day.

Anatomical summary. Gross idiopathic atelectasis both lungs with very gross congestion of abdominal viscera. Patchy bilateral haemorrhagic infarction of both kidneys.

Case 18. A five month old girl was admitted with a six hour history of convulsions. Intensive chemotherapy failed to prevent death in four hours.

Anatomical summary. Petechial haemorrhages in subserosa of intestine, sub-epicardial and subendocardial tissues, lungs and brain. Fulminant septicaemia assumed but no organism recovered.

A dark reddish-purple area occupied about onequarter of the right kidney and involved the medulla more extensively than the cortex. Everywhere the kidneys were congested, but here blood had escaped and disrupted renal elements. There was, however, no inflammatory reaction and no necrosis. Only post-mortem clot was present in the veins.

These cases resemble the condition described by Oppenheim as haemorrhagic renal infarction, but no fibrin thrombi were present in the glomerular capillaries. Except for case 16 the fatal illness progressed rapidly and dehydration was not a feature. The renal haemorrhages tended to be slightly more diffuse and uniform than in the first group. The blood vessels in the loose peri-pelvic tissues were sometimes distended with agonal thrombi, but the smaller intrarenal vessels were relatively inconspicuous, probably owing to pressure on them by the engorged and swollen kidney. A careful search revealed no ante-mortem thrombi in the renal veins and no emboli in the pulmonary arteries. Cases 15 and 17 were associated with gross venous congestion and in cases 15, 16 and 17 some anoxaemia was probably present owing to the incomplete expansion of the lungs.

\section{Infarction secondary to renal inflammation with or without venous thrombosis}

Few cases of pyelitis or pyelonephritis in infancy come to autopsy and in these extensive thrombosis or haemorrhage is rare. Among four cases of pyelonephritis under one month old and three others under one year, two showed small haemorrhages and in two others there were also a few poorly formed thrombi in the veins. These lesions were not comparable in extent to the inflammatory reaction.

Case 19. A male child died on the eighth day with a three-day history of diarrhoea and vomiting. An extensive bilateral pneumonia was present. In the kidneys there were numerous polymorphonuclear leucocytes distributed very diffusely in the medulla, but most marked in the boundary zone. There were very few in the peripelvic or perivascular tissues and their absence from the pelvis or the renal tubules suggested that few could have been present in the urine. In some areas overdistended capillary channels had allowed red blood cells to escape into the oedematous tissue between tubules, but there was no extensive haemorrhage.

Case 20. The findings in this eight-months old child with a seven-day history differed only in the much more extensive inflammatory reaction, in the less marked haemorrhages and in the absence of pneumonia or any lesion productive of anoxaemia.

Cases 21 and 22. In case 21 , a male aged eight days, the inflammatory reaction was similar to that in case 19 , but there were a few ill-formed thrombi and very little haemorrhage. In case 22 , aged three months and ill for three weeks, there was a very extensive inflammatory reaction, but the venous thrombi were terminal and showed no reactive proliferation of endothelium and many veins, though surrounded by inflammatory reaction, contained no thrombi.

\section{Discussion}

A clinical diagnosis will rarely be possible. Blood cells or hæmoglobin in the urine, with or without a palpable abdominal swelling, is very suggestive, especially in a baby suffering from dehydration and low grade or long continued sepsis. In the cases recorded in the literature, blood-stained urine was a frequent, but not constant finding. Barenberg et al. comment on its absence from their cases, and it was rarely observed in the present series. The chief problem for the pathologist is the exclusion of a primary renal inflammation. The absence of reaction in the peripelvic tissue is of no value, but in pyelonephritis polymorphonuclear leucocytes infiltrate between the tubules and the perivascular tissues often contain oedema fluid and inflammatory cells. This reaction is best seen in the boundary zone and in the outer part of the medulla. Thrombosis and infarction secondary to renal infection could scarcely obliterate, and might even emphasize, this inflammatory reaction. A terminal haemorrhagic infarction without thrombosis might result from the local toxaemia produced by a fulminant pyelonephritis, and then there might be little evidence of the previous inflammation. 
Most workers have either avoided the issue or have assumed that the main renal veins become thrombosed first. In pyelonephritis Schröder maintained that thrombosis occurred first in the smaller intrarenal veins. In eleven of the fourteen cases of idiopathic thrombosis described above, there was definite cellular reaction to the thrombi in the smaller veins. The absence of any comparable reaction in the larger interlobar or extrarenal veins suggests that in these thrombosis is later and probably secondary to the resultant stasis. The unequal involvement of small intrarenal veins and the patchy distribution of congestion and haemorrhage in only one kidney also suggest a primary occlusion of the smaller veins. The main renal veins may be thrombosed first in some extrarenal lesions, such as tumours, and by extension of thrombosis from their non-renal branches, but this must be rare in the newborn.

The factors initiating thrombosis in the renal veins are not known, Though both kidneys were almost always involved there was no association with thrombosis elsewhere. Because of the administrative importance of neonatal sepsis this autopsy material is not entirely unselected, but even so dehydration and the toxaemia of continued, rather than fulminant sepsis seem important. Maternal toxaemia was present in only one case (case 8). These cases justify the exclusion of any form of local infection in the kidney, because, though the lesions differed widely in age, none showed inflammatory reaction. This and other thrombotic complications have been seen sufficiently frequently since the advent of intensive sulphonamide and sulphonamide and penicillin therapy in the newborn to stimulate speculation as to some possible relationship. Chemotherapy may influence the bacterial flora of neonatal sepsis and thus alter the toxins produced, or it may prolong a fatal illness and increase the opportunity for thrombus formation. Eosinophilic debris was present in the larger tubules of the inner zone of the medulla in nearly all these cases. This is not unusual in cases of dehydration, and it had provoked no reaction, except occasional and slight proliferation of the epithelium.

Stasis is an important factor in intra-vascular thrombosis. The intrarenal veins were often grossly dilated. This was probably secondary to thrombosis and there was no congenital defect, but it indicates that they can dilate easily and that stasis can occur. The renal tubules of the newborn have relatively little ability to remove water from the glomerular filtrate. Fluid lost by the glomeruli is not regained from the tubules, and, with a circulation slowed by toxaemia and dehydration, the blood in some of the small veins may become unduly concentrated and stagnant. Quick (1942) from a consideration of the curve of clotting time against concentration of thrombin showed that very small changes in thrombin content greatly affect the speed of coagulation. For the prevention of intravascular clotting he emphasized the importance of a continued dilution of such' thrombin as may be formed. This dilution is dependent on an active blood circulation. It would not be useful to discuss the toxins and other factors which may influence the conversion of prothrombin into thrombin. Perhaps in vitro tests have unduly emphasized the importance of the actual amount of prothrombin. In pyelonephritis the vessel walls may be damaged and thrombin production increased, but the blood flow is also increased and thrombosis is often less extensive than might be expected.

A number of factors determine the development of haemorrhagic necrosis in cases with venous thrombi. The outcome depends on the rapidity with which veins are occluded and on the opportunities for opening up anastomotic channels. Especially in the newborn it is also important to consider the effect of toxins, anoxaemia and other changes in the blood, and the degree of systemic venous congestion. Tissue destruction and haemorrhage will, therefore, not necessarily parallel the extent of venous occlusion. Similar factors, singly or together, must dictate the occurrence of haemorrhagic infarction in cases in which the veins are not thrombosed. The possible effects of bacterial toxins on capillary walls must be considered first, but anoxaemia resulting from congenital pulmonary atelectasis (cases $15,16,17$ ) or pneumonia may also be important. Chemotherapy in the early days of life, perhaps even with an absorbed sulphonamide, may interfere with the establishment of the normal bacterial flora of the intestine and with the production of prothrombin. A gross prothrombin deficiency, secondary to this, erythroblastosis (case 15) or other cause might facilitate damage to capillaries, the escape of blood and the disruption of tubules. Primary haemorrhage into the kidneys or into the walls of the renal veins cannot, however, be held responsible for the thrombi in the veins in the first group of cases.

Thrombi in the arcuate veins must interfere with the venous return of both cortex and medulla. The medulla, however, always showed the most advanced lesions. Though the venulae rectae were packed with red blood cells there was nothing to suggest that this was primary. The medulla may suffer most damage because of poorer anastomoses, a lower pressure gradient for circulation, or because the medullary vessels with their long course, thin walls and lack of support distend and rupture readily.

The occurrence of pale infarcts in the kidney in venous thrombosis (cases 13 and 14) is very rarely recognized, but has been ably discussed by Behr. He suggested, on the results of experimental work on rabbits, that this loss of colour occurs within a few days and results from the release of lysins from the infarcted kidney tissue. Haemoglobin, liberated from red blood cells, is removed probably by passage into the urine which is still formed by surviving glomeruli. Some such mechanism must be responsible and it probably only operates fully when there is little disruption of kidney substance by haemorrhage. When there are confluent haem- 
orrhages tearing tubules widely apart, as in cases 11 and 12, the colour change will be longer delayed or may not take place. The involvement of medulla rather than cortex will usually distinguish such cases from the results of arterial occlusion. When large cortical areas also become involved, especially in both kidneys, it may be very necessary to establish the patency of the arterial channels or, as in case 13, to determine which is the older lesion.

White Franklin (personal communication) points out that haemoglobinuria rather than haematuria is regarded clinically as a suggestive sign of renal infarction due to venous thrombosis. Behr's work would provide an explanation for this finding. In the present cases there was no detailed examination of the urine, but in all suspected cases a spectroscopical, as well as a microscopical, examination would be useful.

A variety of lesions was responsible for death in these cases, and associated sepsis was alone often a sufficient cause. Cases 11 and 14 were representative of the more advanced and older lesions and here blood taken soon after death contained 135 and $180 \mathrm{mgm}$. urea per $100 \mathrm{c.cm}$. This is not an excessively high level. Recent work has emphasized the difficulty experienced by the tubules of the kidney of the newborn in the control of the salt and water balance. Dehydration and damage to tubules will increase this difficulty. A feature of interest, not previously noted, has been the high incidence of non-infected emboli in the lung arteries. This occurred in nine of the fourteen cases with thrombi in the renal veins. Similar emboli have been seen apart from renal venous thrombosis, and other foci suggest themselves, but the frequency and the comparable cellular reaction in both kidneys and lungs are very suggestive. Such emboli, if sufficiently large or numerous might explain sudden death in some infants (e.g. cases 2 and 11).

\section{Summary}

In five newborn infants thrombi formed in the intrarenal veins without producing infarction of the kidney. In another five cases thrombi resulted in: haemorrhage between the tubules of some renal papillae, in two others there was extensive disruption, especially of the medulla, by effused blood, and two cases showed both red and pale venous. infarcts. These cases are compared with four where there was haemorrhagic infarction without. thrombi in the veins, and with four, two of which. were within the neonatal period, where similar, but. less marked, lesions resulted from pyelonephritis.

Thrombi were organized or covered by endothelium in eleven cases and had formed first in the small intrarenal branches. Dehydration, continued sepsis and renal venous stasis, but not renal inflammation, appeared to be important in their aetiology. Emboli in the small pulmonary arteries and sometimes small areas of infarction occurred in the lungs of nine of the fourteen cases of idiopathic renal venous thrombosis.

Thanks are due to Dr. F. M. B. Allen for clinical notes and Professor J. H. Biggart for his advice. Mr. D. McA. Mehaffey for photography and Mr. R. Russell for the preparation of histology.

\section{REFERENCES}

Aschner, P. W. (1927). J. Urol., 17, 309.

Barenberg, L. H., Greenstein, N.' W., Levy, L., and Rosenbluth, S. B. (1941). Amer. J. Dis. Child., 62, 362.

Behr, E. (1938). Arch. int. Méd. exp., 13, 273.

Campbell, M. F. (1937). Pediatric urology, New York. and Matthews, W. F. (1942). J. Pediat., 20, 604

Cruickshank, J. N. (1930). Medical Research Council, Spec. Rep. Ser., no. 145.

Faerber, E., and Bussel, R. (1930). Mschr. Kinderheilk., 48, 304 .

Heller, S. (1923). Z Zbl. Gynak., 47, 1897.

Hepler, A. B. (1934). J. Urol., 31, 527.

Marshall, S., and Whapham, E. (1936). Lancet, 2, 428.

Morison, J. E. (1944). J. Path. Bact., 56, 531. (1945). Ibid., 57, 22

Nordwall, U. (1933). Acta Paediatr., Stockh., 14, 186.

Oppenheim, F. (1920). $\quad$ Z. Kinderheilk, 26, 192.

Petramand, S. (1923). Klin. Wschr., 2, 2244. Quick, A. J. (1942). The haemorrhagic diseases,

Schröder, K. (1926). Virchows Arch., 262, 634. 